\title{
Birth Asphyxia. I. Measurement of Visual Evoked Potential (VEP) in the Healthy Fetus and Newborn Lamb ${ }^{(12)}$
}

\author{
JAMES R. WOODS, JR., VALERIE COPPES, DANIEL E. BROOKS, PEGGY J. KNOWLES, \\ MARLA FREEMAN, VALERIE PARISI, PETER OMARA, AND GARLAND E. MCCARTY

\begin{abstract}
From Department of Obstetrics and Gynecology [J. R. W., V. P.], Clinical Investigation Service [V. C., D. E. B.], and Neurology Service [G. E. M.], Department of Medicine, Letterman Army Medical Center, Presidio of San Francisco, California, and the Department of Biorrheology [P. J. K., M. F., P. O.], Letterman Army Institute of Research,
\end{abstract} \\ Presidio of San Francisco, California, USA
}

\begin{abstract}
Summary
The visual evoked potential (VEP) of five healthy, near-term exteriorized fetal lambs was compared with the VEP obtained from the same animals during the first hour after ventilation. Characteristics of the individual wave components and their relationship to blood pressure, heart rate, and arterial blood gas measurements concomitantly obtained were examined. Results show that the VEP of the healthy fetus is a stable, reproducible signal consisting of four basic components, and that the latencies (msec) and the amplitude $(\mu \mathrm{V})$ of the individual components of the VEP are similar to those of the newborn. In two related experiments, fetal asphyxia of unknown duration was encountered during fetal exteriorization and instrumentation. In the first case, neonatal acidosis persisted despite aggressive resuscitation efforts and was accompanied by an atypical VEP. In the second case, the neonatal VEP obtained after 15 min of resuscitation was markedly depressed despite restoration of acid-base balance but gradually recovered during the remainder of the 1-hr newborn period. In conclusion, the VEP is a reproducible measure of cortical function in the healthy fetus and neonate and may reflect the acute status of cerebral function during fetal asphyxia and neonatal resuscitation.
\end{abstract}

\section{Speculation}

The initial success of neonatal resuscitation after birth asphyxia is predicated upon the response of blood pressure, heart rate and blood gas measurements, yet these measurements indicate only indirectly whether brain damage has occurred during asphyxia, or whether brain recovery is eminent. The visual evoked potential may provide a reproducible measure of the effectiveness of current resuscitation practices.

Birth asphyxia and its sequelae represent one of the most uncertain areas of perinatal research. The immediate success of neonatal resuscitation is predicted by the arterial blood gas, blood pressure (BP) and heart rate (HR) response; however, these measures indicate only indirectly whether brain damage has occurred during asphyxia or if brain recovery is eminent.

Recent advances in fetal electroencephalography $(7,8)$ have broadened our understanding of neural function during labor and delivery. The normal fetal encephalogram (EEG) consists predominantly of $\theta(2.5$ to $5 \mathrm{~Hz})$ and $\delta(0.5$ to $2.5 \mathrm{~Hz})$ waves. It is influenced by maternal medications and does not significantly differ from the neonatal EEG. Fetal EEGs, in which prolonged voltage suppression and sharp waves persist, are associated with low Apgar scores, neonatal depression, and long-term neurologic abnormalities $(7,8)$.
Successful measurement of the fetal EEG suggests that more subtle electrophysiologic measurements of brain function may provide additional information during the perinatal period. The EEG measures spontaneous electrical activity over a wide area of the cortex; as such, it tends to reflect random neurologic events (9), and provides little information regarding the ability of the brain to perform a designated task. The brain electrical activity elicited by sensory stimuli may provide this type of information.

The visual evoked potential (VEP) is a 1 to $20 \mu \mathrm{V}$ cortical signal elicited by a flash of light and recorded by EEG scalp electrodes placed over the occipital regions (visual centers) of the brain. The VEP is extracted from the larger $(20$ to $100 \mu \mathrm{V})$ EEG signals by computer methods in which the cortical signals triggered by the stimulus (flash of light) are averaged. Each time a light is flashed, the resulting cortical electrical response is added to responses triggered by previous stimuli. Ultimately, the more random signals of the EEG cancel each other while the VEP is augmented.

Recent laboratory studies $(1,4,6)$ indicate that changes in the VEP may be used to assess cerebral activity during states of altered cardiovascular or respiratory function. Laboratory application of this noninvasive measure to the study of fetal and newborn brain function under normal and stressed conditions has not been explored. In the clinical setting, it is currently impossible to obtain fetal VEPs. In the laboratory, however, access to the exteriorized fetal lamb permits this type of assessment to be made.

The purposes of the present study are: (1) to describe the components and the reproducibility of the VEP in the healthy fetal lamb; (2) to determine the variability of the VEP among fetuses of comparable gestation; and (3) to relate these measurements to cardiovascular and arterial blood gas changes occurring at birth and in the early neonatal period.

\section{MATERIALS AND METHODS}

\section{GENERAL HANDLING OF THE ANIMALS}

Eight pregnant ewes of mixed breed at 135 to 145 days' gestation (term 145 to 150 days) were separately delivered to our animal care facility $1 \mathrm{wk}$ before the study. Animals were provided water ad libitum, were fed a standard diet, and moved freely within a confined area.

\section{INSTRUMENTATION}

On the day of surgery a ewe was brought to the surgery suite. The maternal carotid artery was catheterized for arterial blood gas measurements after infiltrating the surgical site with $1 \%$ lidocaine hydrochloride (Xylocaine). A catheter was then placed in the subarachnoid space and $0.25 \%$ bupivacaine hydrochloride (Marcaine) was administered to the ewe. Part of the uterus was exteri- 
orized through a midline laparotomy incision and marsupialized to the edges of the abdominal wound. The fetus was delivered through an incision in the uterus and the uterine edges were secured to the loose fetal skinfolds around the umbilicus to protect the umbilical circulation. A saline-filled glove was initially placed over the fetal head to inhibit respiration. All subsequent fetal surgery was done after local administration of $1 \%$ lidocaine hydrochloride (Xylocaine). Polyvinyl catheters were placed in the jugular vein for fluid volume and drug administration and through the femoral artery into the aortic arch for systemic arterial pressure and arterial blood gas measurements. The fetal trachea was isolated under sharp dissection and a sealed tracheostomy was placed for later neonatal ventilation.

The saline-filled glove was then removed and the fetal head dried for electrode placement. The left fetal eye was held open with an infant ophthalmic lid retractor, and saline was intermittently applied to the eye to maintain adequate moistening of the tissues.

The VEPs were recorded between bipolar Grass platinum subdermal needle electrodes (Grass Instrument Company, Quincy, MA) placed $1 \mathrm{~cm}$ lateral to the midline over the right and left occipital regions of the fetal skull and referenced to linked electrodes in the midportion of each ear. Electrode impedance was $3000-5000 \mathrm{ohms}$. The ground electrode was placed in the left front leg. VEPs were obtained by stimulating the left eye once per sec with a plain diffuse light of $10 \mathrm{msec}$ duration (Grass PS2 Photostimulator, intensity 16; Grass Instrument Company) placed 28 $\mathrm{cm}$ above the fetal head at the top of a black styrofoam cylinder. The black cylinder was designed to exclude all external light.

The VEP signals, amplified 15,000 times (Grass Model 7P511 amplifiers; Grass Instrument Company) and filtered through a band pass of 0.3 to $1000 \mathrm{~Hz}$ ( $1 / 2$ amplitude), were monitored on a Grass Model 7 polygraph (Grass Instrument Company) with 60 $\mathrm{Hz}$ notch filters and stored on an Ampex Model 500 FM tape recorder (Ampex Corporation, Redwood City, CA). Averaging was not performed when the signal was contaminated with movement artifact. The amplified and filtered signals recorded in the first $500 \mathrm{msec}$ were sampled at a rate of $1 \mathrm{kHz}$ and averaged with a signal averaging unit (Nicolet MED-80, Nicolet Instrument Corporation, Madison, WI). The average of 50 responses was then plotted on graph paper by an X-Y plotter (Hewlett Packard 7035B, Hewlett-Packard, Palo Alto, CA). Latencies (in msec) were obtained by using the cursor on the oscilloscope display and a digital read-out from the computer. The amplitudes were obtained by placing the two cursors at the maximum positive and negative deflections and recording the $\mu \mathrm{V}$ differences between those two components.

During control and testing periods, systemic BP was continuously recorded on a Beckman 4-channel dynograph (Beckman Instruments, Inc., Fullerton, CA). The HR and mean arterial pressure were derived from the pulsatile systemic artery blood pressure recordings. Arterial (femoral artery) blood gas analyses for $\mathrm{pH}, \mathrm{PCO}_{2}, \mathrm{PO}_{2}$, and base excess were obtained from 1-ml heparinized blood samples placed on ice and performed on an IL813 analyzer (Instrument Laboratory, Inc., Lexington, MA).

\section{PROCEDURE}

After fetal instrumentation, a stabilization period of $15 \mathrm{~min}$ was allowed, after which fetal measurements were made. At the beginning of the neonatal period (time 0 ) the tracheostomy was opened, the umbilical cord was ligated, and the neonatal lungs were manually expanded with an Ambu bag during the first min to assure maximum alveolar patency. The lungs were then ventilated with $40 \% \mathrm{O}_{2}$ by a volume-cycle, pressure-limited respirator at 40 $\mathrm{rpm}$, and $3 \mathrm{~mm} \mathrm{Hg}$ positive end expiratory pressure.

VEP, cardiovascular, and arterial blood gas measurements were obtained from the fetus $10 \mathrm{~min}$ before neonatal conversion $(-10$ min) and at 1, 2, 15, and $60 \mathrm{~min}$ into the neonatal period. At 60 min of age the animal was terminated by intravenous injection of T61 Euthanasia solution.

\section{RESULTS}

In the healthy, term, fetal lamb, the VEP is a relatively simple electrical signal composed of four basic peaks. In a typical fetal VEP, the early components NI (first negative deflection), PI (first positive deflection), and $\mathrm{N} 2$ are followed by a major positive deflection (P2) (Fig. 1). Latency represents the time in msec from the light stimulus to the midportion of a specific peak. Latency to $\mathrm{P} 2$ is indicated. Amplitude was measured as the difference in $\mu \mathrm{V}$ from the maximum positive to the maximum negative deflection. Wave forms appearing later than P2 were not considered in the calculation of amplitude.

The stability of the fetal VEP was noted in one experiment in which, after fetal exteriorization, serial measurements of arterial blood gas, VEP, and cardiovascular values were obtained every 20 min for $1 \mathrm{hr}$. The fetal HR and BP fluctuated between 140 to $170 \mathrm{bpm}$ and 63 to $70 \mathrm{~mm} \mathrm{Hg}$, respectively; the arterial blood gases were 28 to $34 \mathrm{~mm} \mathrm{Hg}\left(\mathrm{Po}_{2}\right)$ and 40 to $48 \mathrm{~mm} \mathrm{Hg}\left(\mathrm{PCO}_{2}\right)$, and the $\mathrm{pH}$ was 7.33 to 7.35 . The fetal VEPs taken during this period are shown in Figure 2.

VEP, cardiovascular and arterial blood gas measurements were obtained from five animals in the fetal state, and, after ventilation and cord clamping, during the l-hr neonatal period. Representative changes in these measurements obtained from one animal as the fetus was converted to a neonate are shown in Figure 3. The VEPs of the five animals during the fetal and neonatal period are summarized in Figure 4. Although the general wave pattern described in Figure I was recognized in all cases, the early components $\mathrm{N} 1, \mathrm{Pl}$, and $\mathrm{N} 2$ showed considerable variation in

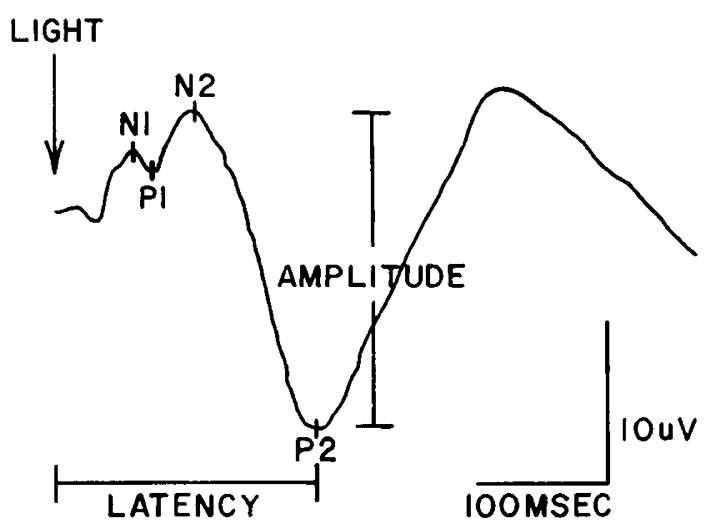

Fig. 1. Typical fetal VEP showing individual components of the wave form and nomenclature used in the study.

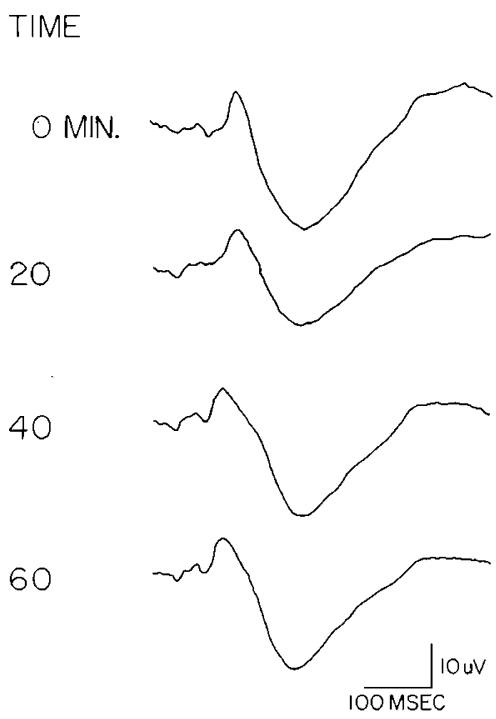

Fig. 2. Stability of the fetal VEP. 
appearance; P2 was the most readily identified component of VEP during the fetal and neonatal study periods, but its appearance also varied considerably among animals.

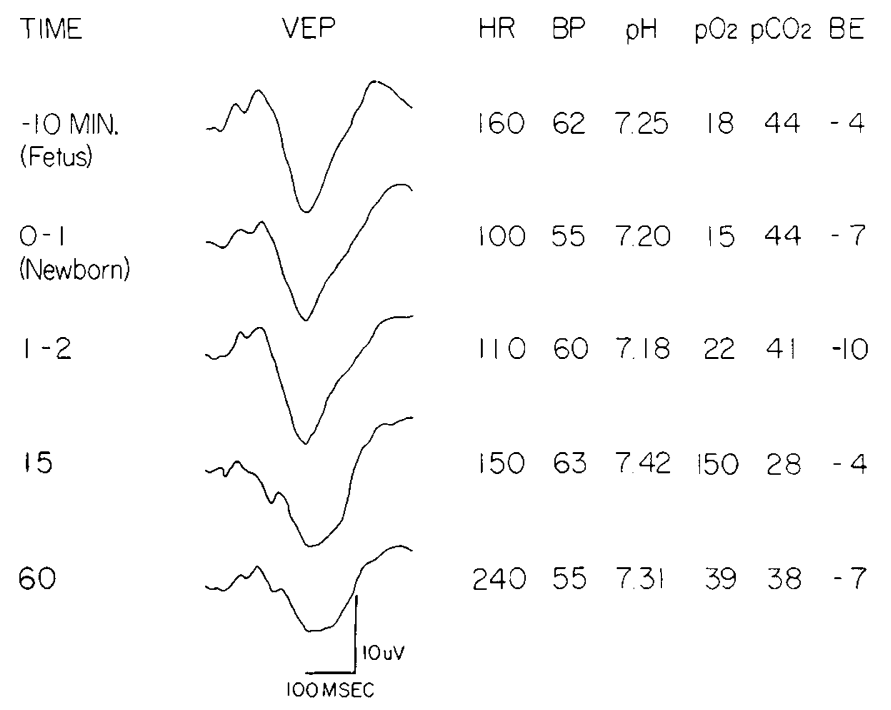

Fig. 3. Typical chances in VEP, cardiovascular and arterial blood gas measurements in the healthy fetus and newborn. $\mathrm{HR}(\mathrm{bpm}) ; \mathrm{BP}(\mathrm{mm} \mathrm{Hg})$; $\mathrm{PO}_{2}$ and $\mathrm{PCO}_{2}(\mathrm{~mm} \mathrm{Hg})$; base excess $(\mathrm{BE})(\mathrm{mEq} /$ liter $)$.
The latencies of the individual components and the amplitude measurements of the VEP obtained from the five animals were grouped in Table 1. During each period of study, latency to the first negative deflection (NI) showed the most uniformity. The latencies of the later wave components, $\mathrm{Pl}$ and $\mathrm{N} 2$, became progressively more variable, and the latency to $\mathrm{P} 2$ consistently showed the widest range of values. The latencies of the individual wave components, however, did not significantly change during the transition from fetus to newborn, and the variations within the latencies remained relatively constant.

Table 1 also shows the cardiovascular and arterial blood gas values which accompanied VEP recordings. During the first few minutes of neonatal ventilation, $\mathrm{HR}$ and $\mathrm{PO}_{2}$ decreased but had little effect upon the VEPs (Fig. 4). With continued ventilation, the $\mathrm{HR}$ and $\mathrm{PO}_{2}$ values quickly recovered.

In two related experiments, fetal asphyxia of unknown duration was encountered during fetal exteriorization and instrumentation. In both instances, immediate neonatal resuscitation consisted of ventilation with $100 \% \mathrm{O}_{2}$ at $50 \mathrm{rpm}$, volume expansion with cord blood and correction of acidosis with sodium bicarbonate. In the first case (Fig. 5), neonatal acidosis persisted despite aggressive resuscitative efforts. The VEP of this acidotic newborn remained atypical during the 1-hr observation period despite an apparent recovery in the $\mathrm{Po}_{2}$. In the second case (Fig. 6), scalp EEG electrodes were placed during resuscitation and VEPs were recorded $15 \mathrm{~min}$ into the newborn period. The VEPs obtained at 15 min were markedly depressed, although at that point resuscitation had restored arterial blood gas, BP, and HR measurements to

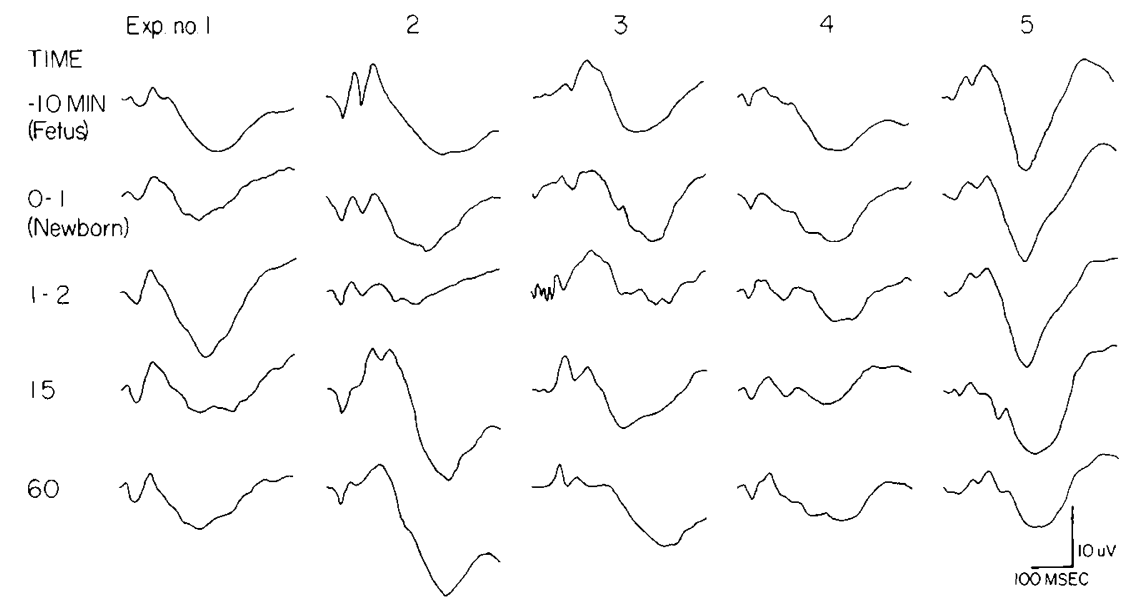

Fig. 4. Comparison of VEPs in 5 animals during the fetal and neonatal period.

Table 1. Components of the fetal and neonatal VEP with corresponding cardiovascular and arterial blood gas measurements

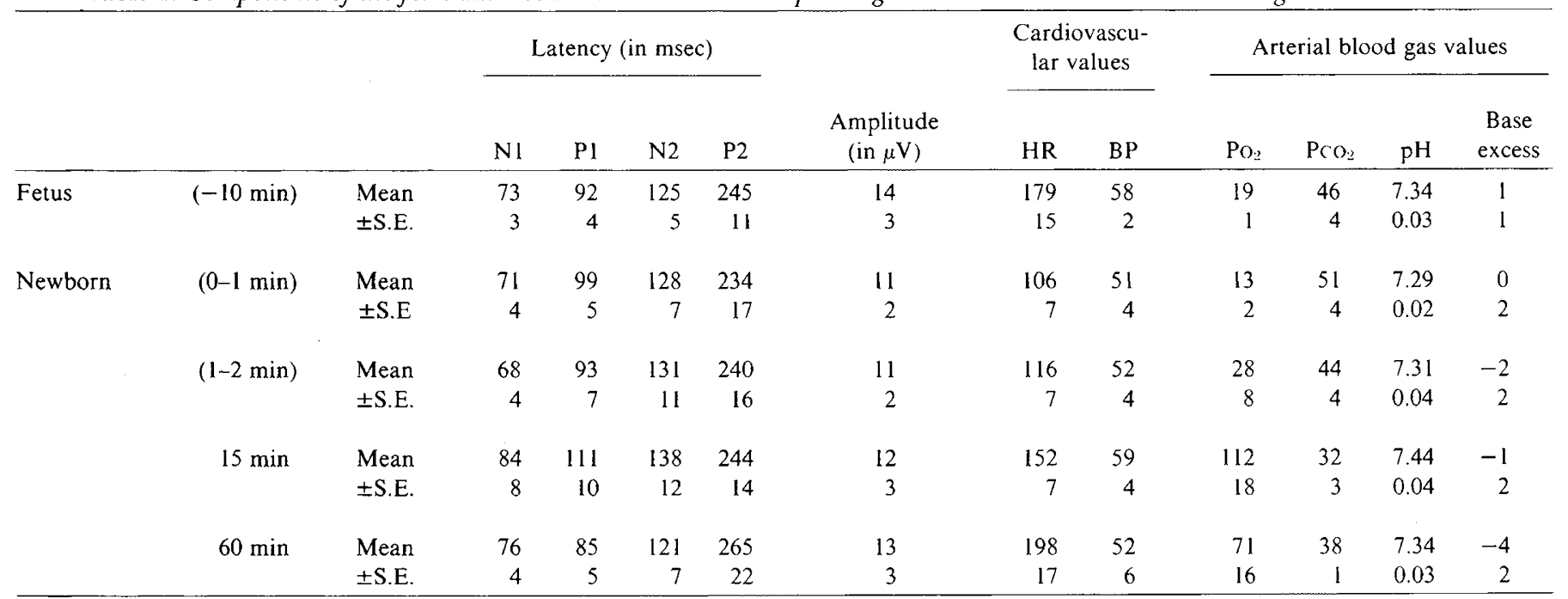

\footnotetext{
${ }^{1}$ Values represent mean \pm 1 S.E. from five animals. $\mathrm{HR}(\mathrm{bpm})$; BP $(\mathrm{mm} \mathrm{Hg})$; $\mathrm{Po}_{2}$ and $\mathrm{PCO}(\mathrm{mm} \mathrm{Hg})$, base excess (mEq/liter).
} 
TIME VEP
INTRAUTERINE
ASPHYXIA
POURATION PDURATION

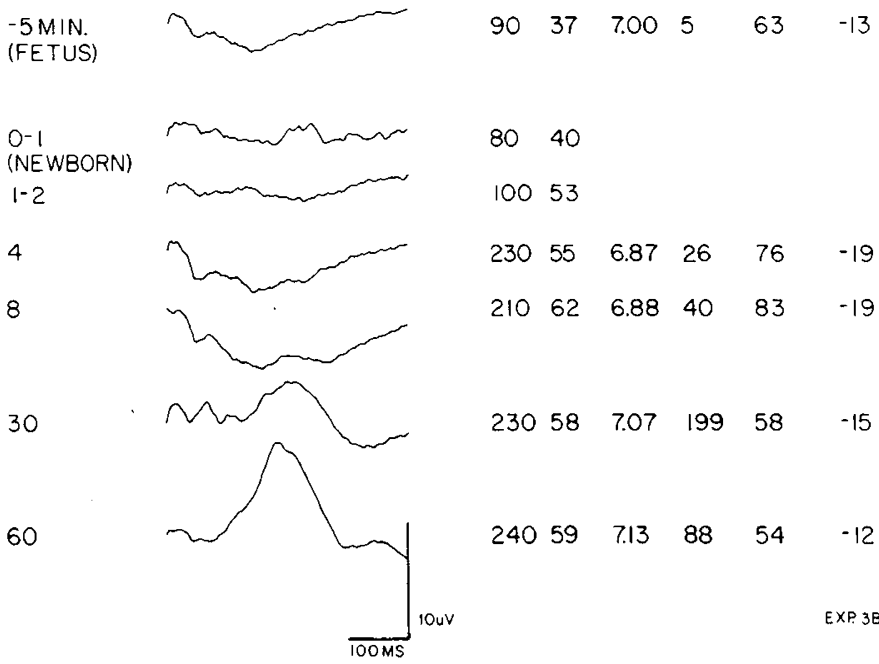

Fig. 5. Example of the effect of fetal asphyxia and neonatal resuscitation upon VEP, cardiovascular and arterial blood gas measurements. HR (bpm); $\mathrm{BP}(\mathrm{mm} \mathrm{Hg}) ; \mathrm{PO}_{2}$ and $\mathrm{PCO}_{2}(\mathrm{~mm} \mathrm{Hg})$; base excess (BE) (mEq/ liter).

TIME
$\begin{aligned} & -10 \mathrm{MIN} \text { (Fetal) } \\ & \text { (Fel) }\end{aligned}$ (Newborn)
20

Fig. 6. Example of the effect of fetal asphyxia and neonatal resuscitation upon VEP, cardiovascular and arterial blood gas measurements. HR (bpm); $\mathrm{BP}(\mathrm{mm} \mathrm{Hg}) ; \mathrm{PO}_{2}$ and $\mathrm{PCO}_{2}(\mathrm{~mm} \mathrm{Hg})$; base excess (BE) (mEq/ liter).

their normal range. During the remainder of the 1-hr newborn period, and while BP, HR, and blood gas measurements remained stable, the VEP gradually recovered.

\section{COMMENT}

It is not surprising that the visual systems of the healthy fetus and newborn respond to a light stimulus in a similar manner. Comparable EEG recordings in the fetus and newborn have previously been noted (8). Because the fetal and newborn VEP are similar in pattern and latency it is suggested that the basic physiologic needs for neural transmission within the visual system are being met in both.
The similarities in VEP of the fetal and newborn lamb also imply that maturational events previously observed in the neonatal and infant VEP $(2,3,5)$ are fetal in origin and continue after birth in association with progressive myelinization of the optic tract. Rose et al. (5) report that VEP obtained in the kitten at 2 to 3 days of age consists of a simple monophasic negative wave. Similar patterns are found at 8 to 10 days in the rat and rabbit, 1 to 3 days in the dog, and at birth in the monkey and premature infant (5). The VEP of the kitten rapidly matures into the more complex wave pattern of the adult in 26 days. The human VEP becomes stable by 9 to $13 \mathrm{wk}$ but only reaches the adult pattern by 18 to 24 months (2).

Recent studies suggest that, like the EEG, the VEP is affected by factors that alter nerve conductivity. Shelburne et al. (6) produced alveolar hypoxia in rhesus monkeys and showed that a $\mathrm{PO}_{2}$ of $18 \mathrm{~mm} \mathrm{Hg}$ or lower significantly suppressed the VEP. Nagao et al. (4) perfused anesthetized baboons with $6 \%$ molecular weight Dextran in order to reduce the hematocrit and thereby impair oxygen transport to the brain; VEP response was lost when the hematocrit fell below 10\%. Kayama (1) demonstrated that, in adult cats, 8 min of alveolar hypoxia eliminated the VEP response to a standard light stimulus, and reappearance of a normal VEP 10 to $20 \mathrm{~min}$ after hypoxia constituted successful resuscitation.

The effect of oxygen deprivation upon VEP of the newborn appears similar to that of the adult $(1,4,6)$. That return of cortical function occurs after recovery of $\mathrm{HR}, \mathrm{BP}$, and blood gas values and may be further delayed by persistent acidosis emphasizes the limitations of clinical monitoring during neonatal resuscitation for birth asphyxia. Since these studies were only carried out to the end of the first neonatal hr, our preliminary data shed no light on the relationship of the VEP to survival or quality of survival. Nor do these observations indicate how well the fetal or neonatal VEP reflect biochemical or electrophysiologic events occurring within the remainder of the brain. Nonetheless, with continued investigation, the VEP may provide a reproducible measure of the effectiveness of current resuscitation practices.

\section{REFERENCES AND NOTES}

1. Kayama. Y.: Evoked potentials of the central visual system during and after hypoxia in cats. Electroencephalogr. Clin. Neurophysiol.. 36: 619 (1974).

2. Evoked potentials. In: R. C. H. Engle: Abnormal Electroencephalograms in the Neonatal Period. Chap. 13. pp. 106-118. (Charles C Thomas, Springfield. IL. 1975).

3. Mattsson, J. L., Fry, W. N., Boward, C. A., and Miller, E.: Maturation of the visual evoked response in newborn miniature pigs. Am. J. Vet. Res.. 39: 1279 (1978).

4. Nagao, S., Roccaforte, P., and Moody, R. A.: The effects of isovolemic hemodilution and reinfusion of packed erythrocytes on somatosensory and visual evoked potentials. J. Surg., Res., 25: 530 (1978).

5. Rose. G. H., Grueneu, S. P., and Spencer, J. W.: Maturation of visual electrocortical responses in unanesthetized kittens: Effect of barbiturate anesthesia. Electroencephalogr. Clin. Neurophysiol., 33: 141 (1972).

6. Shelburne, S. A., McLaurin, A. N., and McLaurin, R. L.: Effects of graded hypoxia on visual evoked responses of rhesus monkeys. In: $R$. L. McLaurin: Head Injuries, Chapt. 12. Chicago Symposium on Neural Trauma (Grune and Stratton. New York, 1975).

7. Rosen, M. G.. Sokol, R. J., Chik, L.. Timor, I., Hertz. R. H., Zador, I.: FEEG and organization of fetal brain. In: L. Gluck: Intrauterine Asphyxia and the Developing Fetal Brain. (Year Book Medical Publishers, Inc., New York. 1978).

8. Sokol, R. J., Rosen, M. G., and Chik, L.: Fetal electroencephalography. In: R. W. Beard, P. W. Nathanietsz: Fetal Physiology and Medicine. (W. B. Saunders Co., Ltd., London, 1976).

9. Sokol. S.: Visual evoked potentials: theory, techniques and clinical application. Surv. Ophthalmol.. 21: 18 (1976).

10. The opinions or assertions contained herein are the private views of the authors and are not to be construed as official or as reflecting the views of the Department of the Army or the Department of Defense.

11. The present address of Dr. J. R. Wood, Jr., is: Department of Obstetrics and Gynecology, University of Cincinnati, Cincinnati, OH.

12. Requests for reprints should be addressed to: Technical Publications Editor, Letterman Army Medical Center. Presidio of San Francisco, CA 94129 (USA).

13. This research was supported by Clinical Investigation Grant 0B-79.

14. Received for publication September 16, 1980

15. Accepted for publication February 2, 1981. 